\title{
Research and Design on Virtual Experiment System Integration Based on WebService
}

\author{
Xuezhen Niu, Fuan Wen, Yanlian Sun \\ Beijing Key Laboratory of Network System and Network Culture, Beijing University of Posts \\ and Telecommunications, Beijing, China \\ Email: douxiaoniu@google.com
}

Received November 2013

\begin{abstract}
With awareness of information teaching being enhanced, related technology and products on virtual experiments have also been continuously developed and improved. However, due to multiparty systems applied result a lack of unity and standardization for experimental teaching processes during the current experimental teaching. This paper purposes integration solutions based on WebService, on the basis of research and analysis of system integrates design, implementation, and virtual experiment platform. The author focuses on realizing integration of Multi virtual experiment teaching resources. Meanwhile through actual trial, results implied that virtual experiment teaching work can be more efficient and normative in university with a standardized process for experiment and management, which can provide a favorable platform basis to achieve resources sharing on virtual experiment in the future.
\end{abstract}

\section{Keywords}

Multi-System; Virtual Experiment; Integration; WebService; XML

\section{Introduction}

With increasing demand of virtual experiment, integration of multi virtual experiment system has become an inevitable trend. As difference of inter-system frameworks and languages, compatibility among systems presents a challenge. Meanwhile we have to access data in disparate systems through different ways [1]. It has caused problems whether from the investment, maintenance, as sharing of data and data redundancy [2]. Therefore, how to achieve the integration of multi virtual experiment resources in a platform has become an inevitable trend.

IM (Information Manifold) is carried out by AT\&T Bell Labs project, used in more than 100 integrated information sources [3]. The University of Southern California Information Sciences Institute developed an integrated system IMS, characterized by the development and application of a variety of artificial intelligence techniques for accessing all kinds of information sources and construct an intelligent dynamic interface [4,5]. Chongqing University's virtual experiments teaching universal platform, through the introduction of next-gen- 
eration applications RIA framework Smart Client, achieves versatility and inheritance of virtual experiment platform to certain extent [6]. Dalian University of Technology iLab is based on the integrated environment Labview Laboratory Virtual Instrument and the structure and build interactive iLab [7].

Currently few studies related to the virtual experiments system integration, as the difference between different virtual experiment platforms [8], so that common, standardized virtual experiment platform integration solutions are difficult to achieve. This is the main point this paper focuses on.

\section{Integrated Technical Overview}

\subsection{Web Service}

\section{Web Service Overview}

Web Service is a platform-independent, loosely coupled, self-contained, programmable based on web and XML standards, and it can describe applications [9].

Web Services is a distributed technology using XML as the basic data communication, so heterogeneous systems can be used as part of collaborative computing network operation [10]. Its main objective is to present a common platform-independent, language-independent platform, with a standard Web protocol providing services to achieve the kinds of applications on different platforms connectivity and integration [11]. Web service can be described to publish, found, and called network and information systems. And it can provide a faster rate to access cross-platform data services, and achieve integration of all data on server, successfully resolved the problem of heterogeneous data using various systems $[12,13]$. Web service works is shown in Figure 1.

\subsection{XML}

XML (Extensible Markup Language) is a markup language and the Standard Generalized Markup Language (SGML) subset for Web traffic. XML is the universal format for data exchange; as a key technology of WebService, it is mainly used as a document, XML provides a uniform method to describe and exchange independent structured data of applications or vendors.

XML can define the management information structure in a flexible and effective way. XML can be defined in a flexible and effective management information structure, the data stored in XML format not only have good internal structure, but also because it is an international standard proposed by the $\mathrm{W} 3 \mathrm{C}$, and thus supported by the majority of software providers for easy data exchange and development.

\section{Integrated Program Based on WebService}

The virtual experiment in this paper is based on WebService and is developed using JAVA. Which is B/S mode and has a SSH architecture, and has certain advantages in terms of flexibility and scalability.

\subsection{Platform Integration Solutions}

The integration solution is as shown in Figure 2.

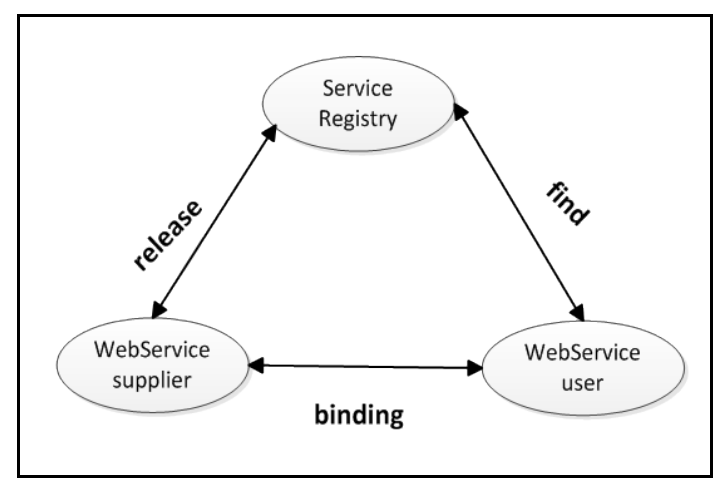

Figure 1. Webservice works. 


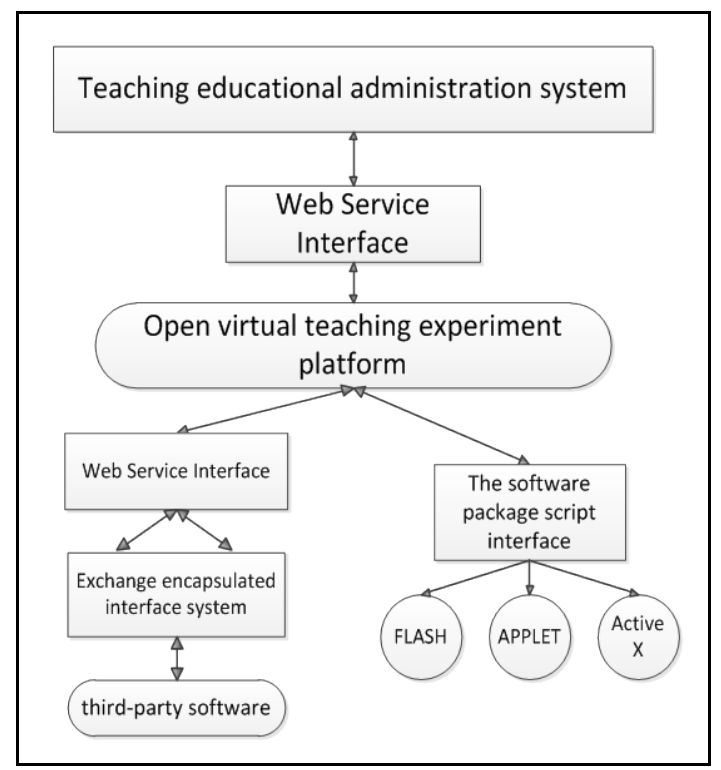

Figure 2. The integration solutions

1) Integration with teaching educational system:

First part is the user's butt, using single sign-on technology, unified entrance for the school comprehensive educational system, while the user information automatically imported into virtual experiment teaching system. Second part is the selecting and scheduling module which can directly into the virtual experiment teaching system and can be rescheduled, this function uses Web Service technology. Third part is data transformation.

2) Integration with the third party standardized virtual experiment system

The third-party software system works as a virtual experiment teaching system service. Virtual experiment teaching system calls the services provided by third-party software systems to complete the experimental teaching, WebService used in the experiment teaching process.

Integration configuration: Senate login virtual experiment teaching system management course information, and configures the third-party software system integration interface configuration.

Experiment maintenance integration: First, integration on existing experiment database, third-party provides experiment extracted service interface for synchronization. Second, edit interface services, when teacher adds or modifies experiments, platform invokes third-party services for editing, and then experiment libraries updated.

Students' doing experiments Integration: Student logins system, and starts the experiment. Then service ID is generated and third-party software systems interface is called, students experiments page displays, after finishing, third-part treats service ID as identification, then students' results are saved and returned.

Teachers marking experiments integration: Teachers log into arrangements list, select what they want to modify, then platform calls third-party interfaces based on the service ID system service, student results display.

3) Integration with third party standardized virtual simulation software

Integration with third-party virtual simulation software, such as: Flash, Applet, ActiveX and other software. Integrated into the virtual experiment teaching system in a form of an experimental plug-in, improve the management mechanism with the help of virtual experiment teaching system, and get use to the advantages of the third-party virtual simulation software simulation, and complete the experimental teaching process.

\subsection{Integration Interface Architecture}

Platforms and virtual experiment system and teaching-related systems communicate by passing XML file, and integrated interface provides a unified data format, standardized communication between systems.

After different data sources processed by the Web Service, the obtained data is saved in xml format the data, and then through the XML data integration processing module transform the xml data into a unified format specification data files, back to the current call virtual experimental system, by that xml parsing system, return the results to the user. The overall framework of integrated design is as shown in Figure 3. 


\section{Achievement of Integration Based on the WebService}

\subsection{Experimental Process}

The specific process as shown in Figure 4.

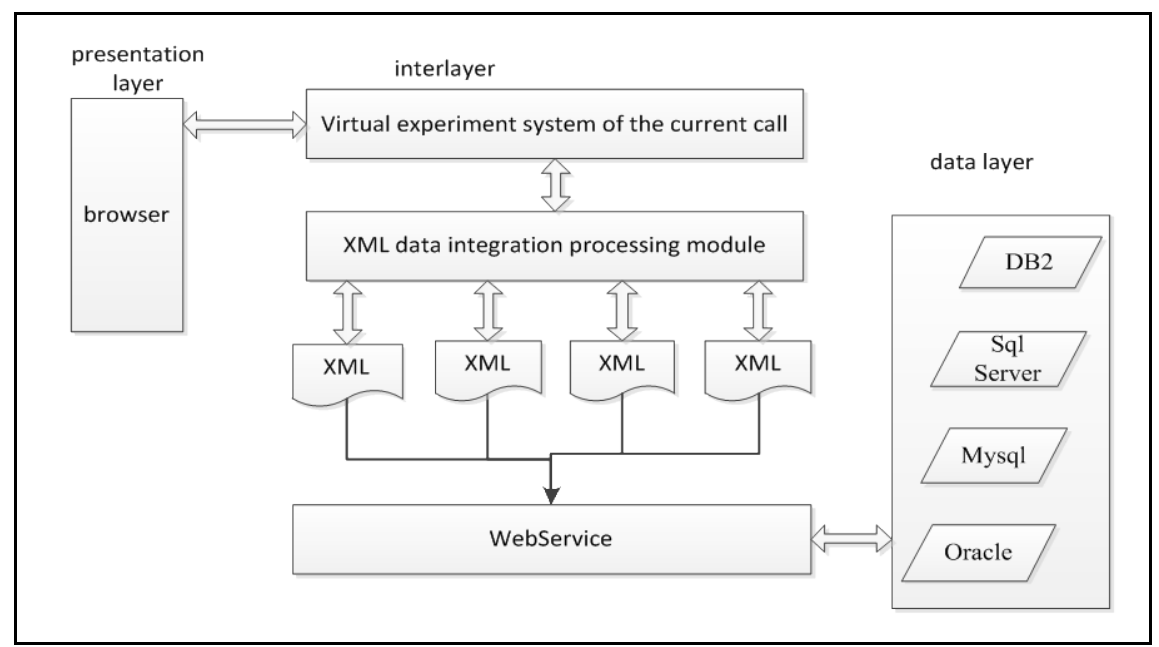

Figure 3. The overall framework of integrated design.

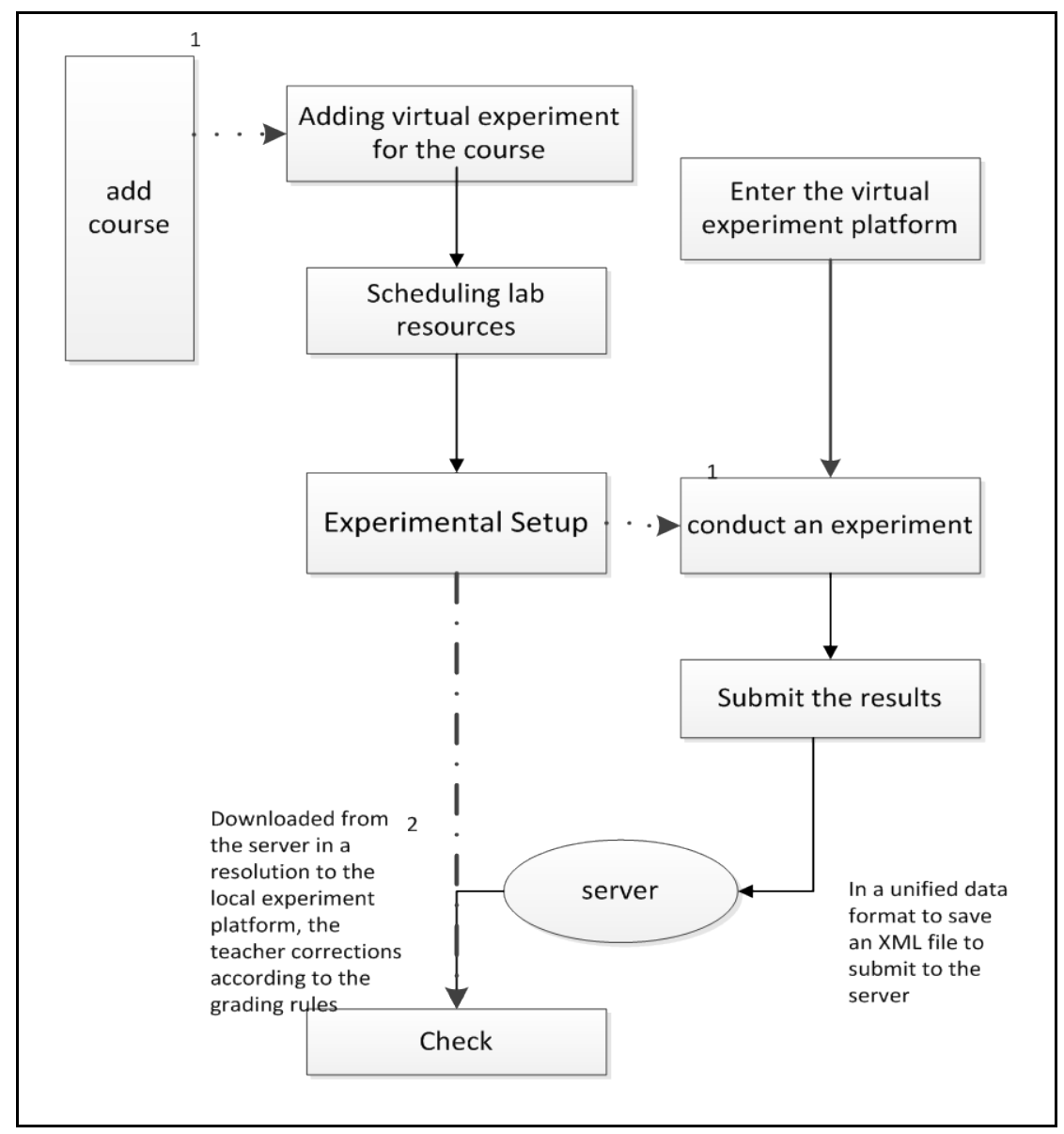

Figure 4. The specific process. 
1) Add academic courses: Academic staff add courses, and select the corresponding interface, to achieve multi-platform integration.

2) Teachers add experiment: Teachers add virtual experiments according to the arrangements; they can select the required third-party plugins, and then add rules and report templates.

3) Teachers arrange the experiment: Teachers arrange the experiment according to the progress of the class.

4) Students do experiments: Students enter the system, they can view the arrangement. They first complete the corresponding exercises, then enter the virtual experiment platform and complete the experiment.

5) Save experimental results: After submitting, the experimental results are saved with XML files and in unified standard format. These data have to be transformed as they come from different servers with different format.

6) Teachers marking experiment: Teachers choose students marked, and download to local through the parse of the third-party systems.

\subsection{Integrated Interface}

1) Integration with the third party standardized virtual experiment system

- Web Service service wsdl address: Experimental repository synchronization interface (third-party systems). Web Service method for third-party to provide synchronization experiments: synchroNAMEExps ().

- Design of experiments callback platform interface (experimental teaching platform); Experimental teaching platform provides Web Service method updates the library experiment experiments: saveExp (parameters), send the information to add or modify experimental XML data.

- Teachers design an experimental interface (third-party systems): Teachers design an experimental interface path and the incoming service ID\& third experiment ID

- Do experiments Interface (third-party): Experiment interfaces path, incoming service ID \& third experiment ID

- Students submit results Interface (experimental teaching platform): Experimental teaching platform provides Web Service method updates the students experiment results: saveStudentExpScore (parameters) to send students to experiment results XML data.

- Check the specific steps students experiment (third-party systems): The third-party systems provide We Service method to view student experimental steps: viewStudentExpInfo (serviceId), specific steps to return students to experiment XML data.

2) Integration with third party standardized virtual simulation software

The third-party software vendors to provide access to platform scripting interface: getPlatFormXml ().

- Third-party software vendors to provide an interface to load the initial script: initPlatFormXml ().

- The third-party software vendors script standards.

\section{Conclusions}

After completed platform integration interface design and implementation, the classes added to the system based on the integrated type and select interface, as shown in Figure 5.

While selecting the local programs, the third-party plug-ins can be selected according to the experimental test requirement, as shown in Figure 6.

Selecting third-party program set the third experimental system structure shown in Figure 7.

The design of the integrated interface makes the virtual experiment platform integrated with multiparty systems and plug-ins. Meanwhile, it achieved the asynchronous refresh and strengthens the effect of the use of the platform by using AJAX, JavaScript.

But now there are many deep-seated problems to solve. Web Service the efficiency, the use of xml language, can achieve platform independence, but using xml transmission efficiency is always a problem, which still needs follow-up optimization.

\section{课程类型 $=\bigcirc$ 第三方课程 $\odot$ 本地课程}

Figure 5. The integrated type and select interface. 


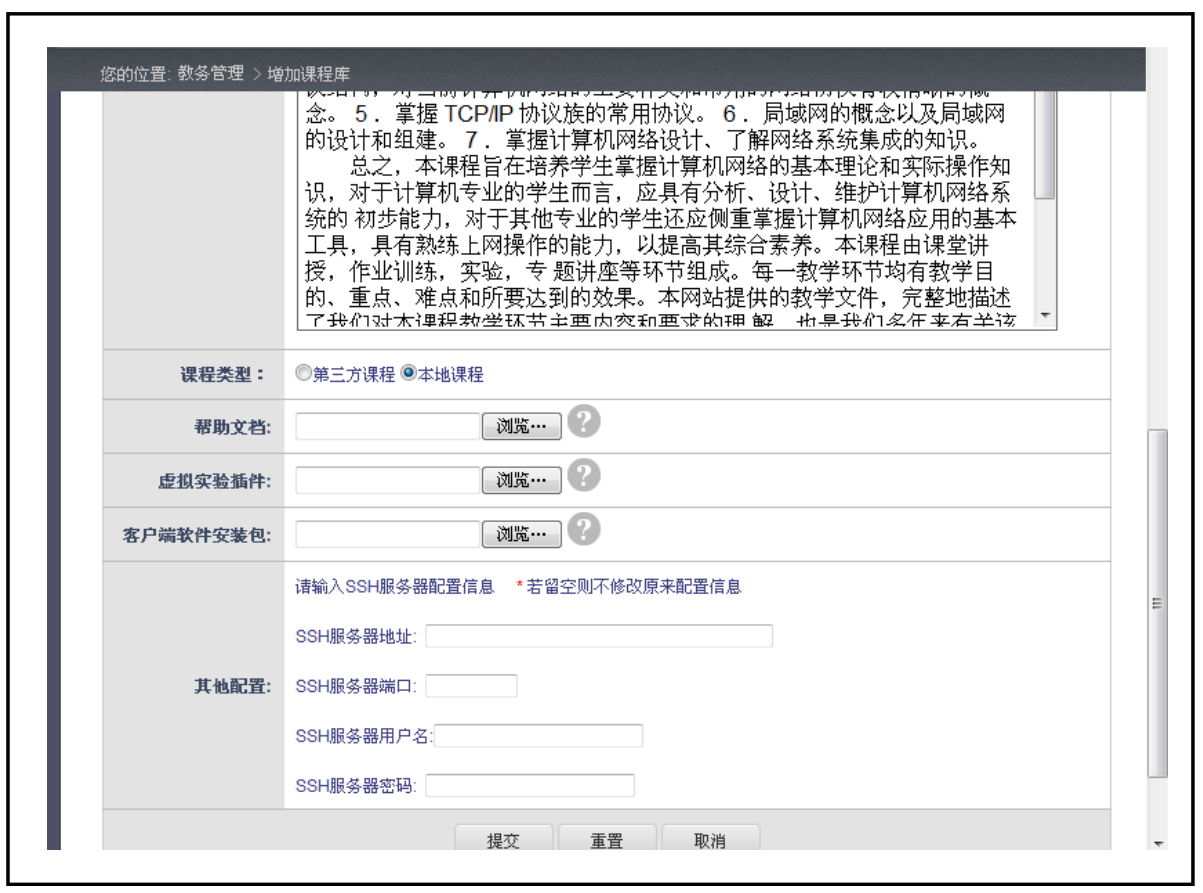

Figure 6. The experimental test requirement interface.

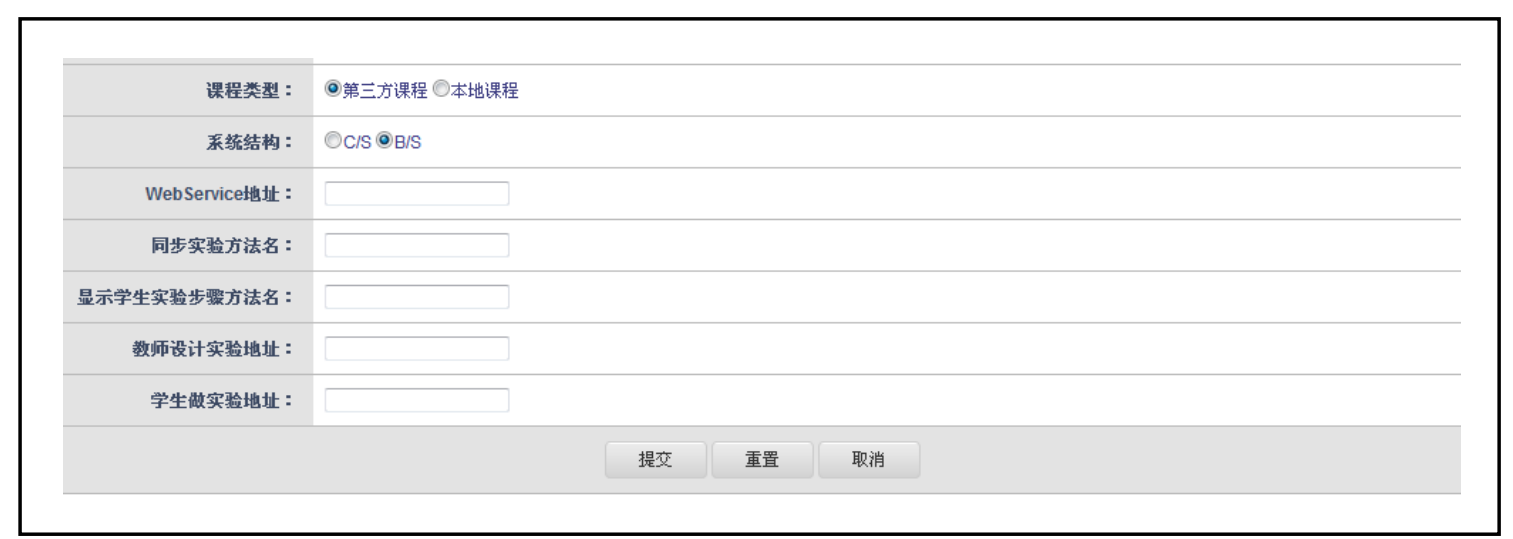

Figure 7. The third experimental system structure.

\section{Comment}

The Special Foundation for Scientific and Innovative Project of BUPT, Research and development of the virtual experiment system on robotics.

\section{References}

[1] Xie, B.Z. and Wang, L.K. (2010) Data integration middleware based on XML and WebService. IT, 1, 90-91.

[2] Chou, M. and Zhu, X.L. (2011) A survey of school information system integration based on WebService. Ningbo Vocational and Technical College Journal, 15, 49-50.

[3] Arens, Y., Knoblock, C.A. and Chen, W. (1996) Query reformulation for dynamic information integration. Journal of Intelligent Information Systems, 6, 99-130. http://dx.doi.org/10.1007/BF00122124

[4] Arens, Y., Chee, C.Y., Hsu, C., et al. (1993) Retrieving and integrating data from multiple information sources. Journal of Intelligent and Cooperative Information Systems, 2, 127-158. http://dx.doi.org/10.1142/S0218215793000071

[5] Xu, T. (2006) Research and application on common platform for virtual experiment teaching-oriented. Chongqing Uni- 
versity.

[6] Gao, F. (2008) Research on network laboratory based on lab view [D]. Dalian University of Technology.

[7] Shi, D.Q. (2011) Research on framework of school integration application system based on tuxedo and webservice. Silicon Valley Technology Develop, 21, 87.

[8] http://baike.baidu.com/view/67105.htm?fromId=837392

[9] Wang, Y., Cai, G.Q. and Wang, X.W. Application of system integration technology in network education platform based on webservice.

[10] Zhou, H.G., Chen, W.G. and Liu, H.J. (2007) Research on integration solution for enterprise information management system based on WEB services. Electrical Engineering Technology, 36, 43-44.

[11] Bian, H.L. (2009) Research and achieve of transmission data corresponding to webservice. Computer Knowledge and Technology, 44, 1644-1646.

[12] Zhang, L.G., Liu, Y. and Chen, Y.Y. (2008) Design and achieve of data communications integrated network management system. Computer Application and Software, 48, 133-135, 144.

[13] Miao, Q. (2008) School application integration based on webservice. Computer Technology and Develop, $3,18$. 